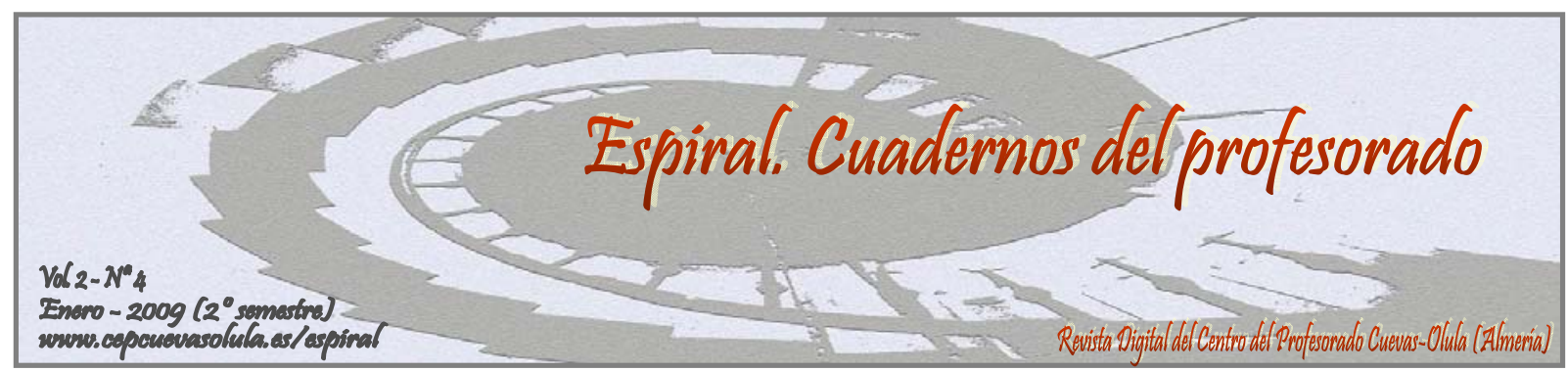

\title{
EL ENTORNO NATURAL Y LAS ACTIVIDADES FÍSICAS EN EL MEDIO NATURAL COMO BASE PARA UN PROYECTO EDUCATIVO MULTIDISCIPLINAR
}

\section{NATURAL SURROUNDINGS AND PHYSICAL ACTIVITIES AS A BASIS TO A EDUCATIVE PROJECT TO MULTIDISCIPLINE}

\author{
Manuel Beas Jiménez, Francisco Rodríguez Prados \\ y Andrés Jesús Martínez Moreno
}

IES Cerro Milano de Alhama de Almería, Almería, España

\begin{abstract}
RESUMEN: El aprovechamiento del entorno natural, del que disponen los centros educativos de zonas rurales, para la práctica docente, se convierte en una herramienta pedagógica de enorme interés debido a la gran cantidad de contenidos que se pueden trabajar en él. Las Actividades Físicas en el Medio Natural (AFMN) serán el medio para acercarnos al entorno, desplazarnos e interaccionar con él, permitiéndonos un trabajo interdisciplinar que conexiona multitud de áreas del conocimiento. De esta forma se busca un proceso de enseñanza-aprendizaje más significativo y motivante para el alumnado, trabajando de forma coordinada, un total de 12 departamentos docentes, durante tres momentos pedagógicos (antes, durante y después de la ruta), a través de un proyecto educativo de carácter multidisciplinar denominado “Cuaderno Multidisciplinar: Itinerario Barranco del Pasillo” el cual incluye un cuaderno del profesorado con orientaciones pedagógicas para poner en práctica la actividad y un cuaderno del alumnado con diversas propuestas de actividades en cada uno de los momentos pedagógicos citados.
\end{abstract}

Palabras clave: Actividades Físicas en el Medio Natural, entorno natural, práctica docente, interdisciplinariedad, proyecto educativo.

ABSTRACT: The advantage of the natural surroundings for the teaching practice by educative centres in rural areas turns into a pedagogical tool due to the great amount of contents that can be worked there. Physical Activities at Natural Surroundings (PANS) will be a key tool to approach to our environment, to move inside it and interact with it, allowing us a multidiscipline teaching work, that links up a lot of knowledge areas. In this way we look for a more significative teaching-learning process, more motivating for the students, working for a common purpose twelve teaching sections, during three pedagogical moments (before, during and after the route), through a multidiscipline educative project called "Multidiscipline Exercise book: Itinerary Barranco del Pasillo". This includes a teacher exercise book with pedagogical advices to carry the teaching activity out and a student exercise book with different activities proposals in each one of the mentioned pedagogical moments.

Key words: Physical Activities at Natural Surroundings, environment, teaching practice, educative project. 
Beas Jiménez, M., Rodríguez Prados, F. \& Martínez Moreno, A.J., B. (2009). El entorno natural y las actividades físicas en el medio natural como base para un proyecto educativo multidisciplinar. Espiral. Cuadernos del Profesorado [en línea], 2(4), 56-65. Disponible en: http://www.cepcuevasolula.es/espiral.

Fecha de recepción: 22/04/2009

Fecha de aceptación: 17/05/2009
Enviar correspondencia a: yobeas@gmail.com

\section{1.- INTRODUCCIÓN. ORIGEN DE LA EXPERIENCIA.}

El presente artículo pretende exponer los principales aspectos del proyecto educativo que se está llevado a cabo en el IES “Cerro Milano" de Alhama de Almería, cuyo principal objetivo es el aprovechamiento del entorno natural de las inmediaciones del centro educativo para la práctica docente. Empleando dicho entorno y las actividades físicas en el medio natural (AFMN) como nexo de unión entre las diferentes materias que participan en el proyecto, se ha elaborado un material pedagógico que plantea muy diversas actividades, de carácter interdisciplinar, que se recogerán en un cuaderno del profesorado y otro del alumnado, los cuales serán publicados en breve.

El proyecto educativo que presentamos se basa en un proyecto anterior, denominado Itinerario Geológico por el Barranco del Pasillo, elaborado gracias a la colaboración entre nuestro centro educativo, la ADR (Asociación para el Desarrollo Rural) Alpujarra Sierra Nevada y la empresa de agentes estratégicos medioambientales Ecomímesis. Dicho proyecto culminó con la publicación de una guía didáctica, una guía turística y un cuaderno geológico escolar que permiten conocer este increíble espacio natural de la localidad de Alhama de Almería.

Sin embargo, por las características del proyecto, la guía didáctica se vio limitada exclusivamente a los aspectos relacionados con la geología de este singular paraje natural, que incluye alguna formación geológica, catalogada por la Junta de Andalucía, como bien de interés geológico.

Es por ello que surge un grupo de trabajo, formado por un total de doce departamentos diferentes $y$ unos quince miembros del profesorado, coordinados por el departamento de Educación Física del centro, que elaboran un nuevo proyecto pedagógico, cuyo principal cometido consiste en desarrollar una serie de materiales didácticos, de las diferentes áreas participantes en dicho proyecto.

\section{2.- OBJETIVOS.}

Tras una labor docente de varios años, en nuestro centro educativo, empleando de forma habitual el "aula naturaleza", en la asignatura de educación física, el reciente grupo de trabajo se plantea la posibilidad de abordar este tipo de actividades de una forma interdisciplinar, dadas las increíbles posibilidades que la naturaleza ofrece en este sentido y los enormes recursos pedagógicos que el entorno natural posibilita al centro educativo.

Así pues, los principales objetivos que se pretenden conseguir con el proyecto, muchos de ellos coincidentes con los del proyecto anterior, en el cual se basa, son los siguientes:

$\square$ Abrir el centro a su entorno.

$\square$ Aprovechar los espacios naturales que ofrece dicho entorno, a los centros educativos rurales, como recursos didácticos.

$\square$ Motivar al alumnado, captando su atención, mediante aprendizajes significativos, basados en la aplicación de conocimientos en situaciones y contextos reales.

$\square$ Generar documentación, de carácter pedagógico y multidisciplinar, para su utilización por parte de los centros educativos de la localidad, así como por parte de otros centros, para una enseñanza sensibilizada con el uso y disfrute de nuestros entornos naturales, basada en el respeto y conservación de dichos entornos.

\footnotetext{
${ }^{1}$ Nombre con el que Aguado, M. (2001), define el entorno natural cuando se utiliza como espacio docente.
} 
Colaborar con otras instituciones de cara a publicar y difundir dicha documentación.

\section{3.- FUNDAMENTACIÓN.}

Podríamos definir las AFMN como aquellas actividades de marcado carácter motriz que realizamos en el medio natural con un propósito educativo (Aguado, 2001).

En la nueva cultura posmoderna, la búsqueda de placer, la diversión, la exaltación del presente, se valen del ocio como elemento perfecto para la transmisión de los diferentes valores de dicha cultura. Así pues las diversas prácticas para la ocupación del ocio pasan de ser un elemento de segundo orden a incorporarse, con fuerza, en nuestras vidas, como oportunidad para nuestra realización personal y el desarrollo de nuestra propia identidad. (Águila, 2007)

En la tremenda vorágine de oferta, consumismo e información que nos ofrece esta nueva sociedad posmoderna, aparecen diferentes prácticas o actividades físicas, en la ocupación del tiempo de ocio, cuyo escenario de práctica es el medio natural.

La práctica docente, entendida como el conjunto de conocimientos que el profesorado pone a disposición del alumnado para favorecer su proceso de enseñanza-aprendizaje, debe contemplar la realidad social del momento incorporándola, en la medida de lo posible, a dicho proceso.

Es por ello que nosotros, los docentes, debemos fomentar en nuestros centros este tipo de actividades como un contenido educativo más, que podemos abordar tanto en el ámbito escolar como extraescolar (Santos, 1999).

En el nivel educativo de Enseñanza Secundaria obligatoria se contempla un bloque de contenidos denominado "La Actividad Física en el Medio Natural" en el cual se trabajan las habilidades motrices básicas propias de cada actividad, con el objetivo de conseguir una autonomía de acción, en este medio, que nos posibilite desplazarnos por él, aprendiendo a valorarlo y respetarlo y tomando conciencia del impacto que estas actividades tienen sobre dicho medio.

Así pues, la docencia, en general y en zonas rurales, en particular, debe aprovechar las enormes posibilidades que le ofrece el entorno en el que se encuentra y lejos de dar la espalda al medio natural, debe saber beneficiarse de los innumerables recursos pedagógicos que éste le brinda.

No es posible educar sólo con los elementos condicionantes del aula; otros elementos externos como la familia, el entorno, el medio... posibilitan una actuación continuada del verdadero sentir y proyección del hecho educativo. Descubrir nuevos parajes, nuevas situaciones personales y sociales en contextos ambientales diferentes a los habituales, aportan un componente educacional de alto valor.

El hecho de salir del centro educativo y desplazarse a un medio natural, durante varias horas o a veces incluso días, da a estas actividades, una característica, normalmente, de novedosas que hace que gocen de un elevado nivel de aceptación por parte del alumnado que, "generalmente, participa en ellas con un grado de motivación intrínseca muy alto"(Aguado, 2001).

Como hemos señalado en otras ocasiones (Beas, 2008) dentro de la práctica de actividades físicas en el medio natural, el senderismo es, sin duda, una de las más asequibles pues apenas se necesita material específico ni una gran condición física, basta con un calzado adecuado, una pequeña mochila para llevar un poco de agua y algo de comida, una gorra o similar para protegernos del sol y ganas, muchas ganas de andar, una de las actividades físicas más naturales en el ser humano y para la cual estamos biomecánicamente mejor preparados y adaptados.

Además el senderismo será, en numerosas ocasiones, el medio de desplazamiento ideal y a veces único para acercarnos hasta los lugares de práctica de otras actividades físicas en el medio natural, convirtiéndose en una de las AFMN más empleadas a nivel educativo.

No vamos a detenernos en exceso en descubrir ahora los beneficios de la práctica de actividades físicas en el medio natural, pero sí que creemos conveniente recordar dos de ellos:

$\Leftrightarrow$ Contribución a la mejora de nuestra salud ya que se trata de actividades que desarrollan nuestra capacidad aeróbica general, nos ayudan a tonificar la musculatura, principalmente del tren inferior (piernas) y desarrollan nuestras habilidades motrices básicas (coordinación, equilibrio, agilidad...) dependiendo principalmente de la variedad de terrenos por los que practiquemos dichas actividades. 
$\Leftrightarrow$ Contacto con el medio natural pues nos acercan a la naturaleza permitiéndonos, durante la duración de la actividad, escapar del medio urbano en el que discurre la mayor parte de nuestro tiempo, disfrutando y conociendo el medio natural, su flora, su fauna, despertando nuestros sentidos con sus sonidos, colores y olores y ayudándonos a liberar el estrés de nuestra vida cotidiana.

No cabe duda que son muchos más los beneficios que nos puede aportar la práctica de AFMN, lo cual unido a las características particulares de este tipo de actividades, diversidad y amplitud de espacios, medio desconocido, espacio cambiante, experimentación de las fuerzas de la naturaleza, diversidad de entornos (próximos, cercanos o lejanos), contextualización de los conocimientos de muchas áreas, diversidad y variedad de estímulos, estimulación de los diferentes sentidos, etc., no hace otra cosa que confirmarnos el enorme interés educativo de las mismas, al que ya hemos hecho referencia anteriormente, y afianzarnos en la idea de su necesaria aplicación e inclusión en los proyectos educativos de cualquier centro de enseñanza.

De esta forma hemos basado nuestro proyecto, tal y como hiciéramos con su antecesor, en dos principios básicos de la enseñanza, la enseñanza activa y la enseñanza emancipatoria. La primera implica la consideración del alumnado como ente activo, que para conseguir soluciones y resultados, debe movilizar sus capacidades, la segunda se refiere a la necesidad de conceder al alumnado un nivel de responsabilidad y capacidad de toma de decisiones, acorde con sus posibilidades (Sánchez Bañuelos, 1986).

Como ya hemos indicado, también, en alguna otra ocasión (Beas et al, 2009) las actividades físicas en el medio natural facilitan sobremanera ambos principios y la consecución de los mismos pues el alumnado que participa en este tipo de actividades, en numerosos momentos, se ve obligado a la toma de decisiones propias lo que desarrolla su nivel de responsabilidad, mientras que por otro lado, la gran riqueza de posibilidades que nos ofrece el medio natural, en las muy diversas prácticas existentes, requieren del alumnado la aplicación de diferentes capacidades tanto motrices como cognitivas concernientes a numerosas áreas del conocimiento.
Partiendo de estas premisas y facilitado por el entorno natural que nuestro centro educativo, enclavado en una zona rural, tiene a su alcance, el grupo de trabajo ha elaborado una serie de materiales, gracias a la colaboración con la empresa de agentes estratégicos ambientales Ecomímesis, que se recogen bajo el título de "Cuaderno Multidisciplinar: Itinerario Barranco del Pasillo".

\section{4.- EL PROYECTO.}

Contando con la implicación de un elevado número de docentes en aspectos como la organización y realización de actividades, la exploración del entorno y la elaboración de proyectos, sin la cual este cuaderno no habría sido posible, se estructuró todo el material didáctico atendiendo a los diferentes momentos pedagógicos, de manera que se presentan una serie de materiales para trabajar desde las distintas áreas antes de la ruta (como preparación de la ruta y desde el ámbito de cada materia), durante la ruta (como realización de la ruta con actividades en el entorno natural, también desde la perspectiva de cada área, pero con un carácter globalizador e integrador de todas ellas) y después de la realización de la ruta (como actividades de valoración de la misma que nos permitan detectar errores e introducir nuevas mejoras).

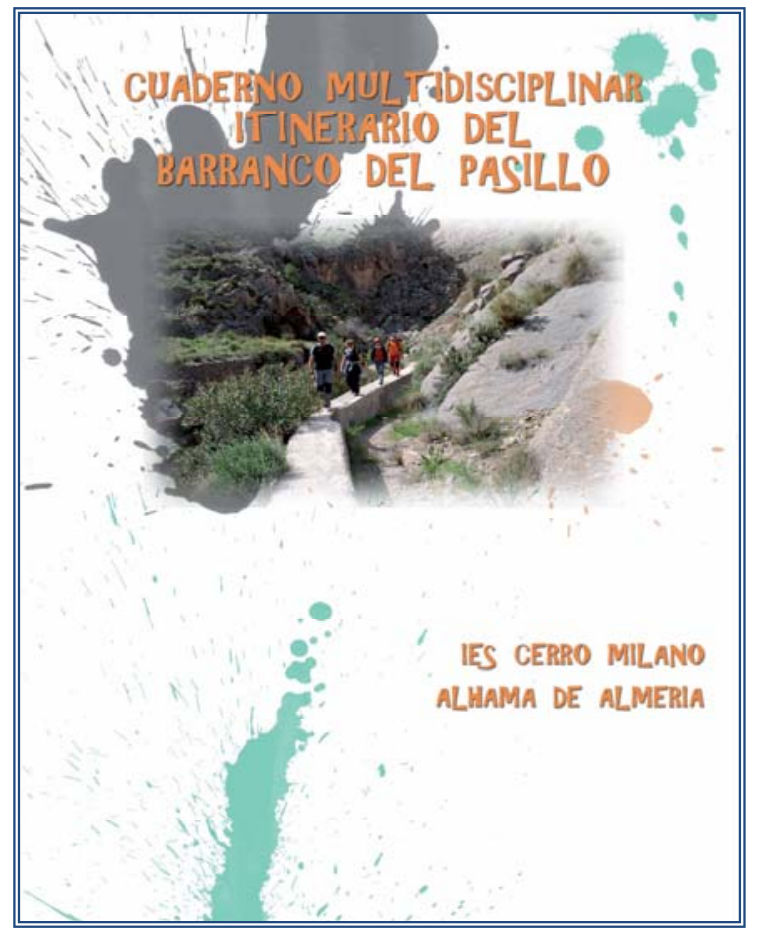

Figura 1.- Portada del cuaderno multidisciplinar del itinerario del Barranco del Pasillo. 
El cuaderno (figura 1) cuenta con varios itinerarios, diferentes para cada nivel educativo, que requieren la práctica del senderismo e incluso, en algún caso, el empleo de técnicas de descenso de barrancos, que nos permitirán conocer con detalle este bello paraje natural.

Como ejemplo de esa interdisciplinariedad mostramos las actividades de algunos departamentos, destinadas a $4^{\circ}$ de ESO para Matemáticas y $3^{\circ}$ de ESO para Ciencias Sociales. A continuación se presentan una serie de actividades, de cada una de estas materias, basadas en los contenidos y que, teniendo como referencia los objetivos que la legislación marca en relación al curriculum, evidencian la relación existente en nuestro cuaderno multidisciplinar, con el medio natural por el que discurre dicho itinerario así como con las actividades físicas en el medio natural que nos permiten disfrutar del mismo, el senderismo y el descenso de barrancos.

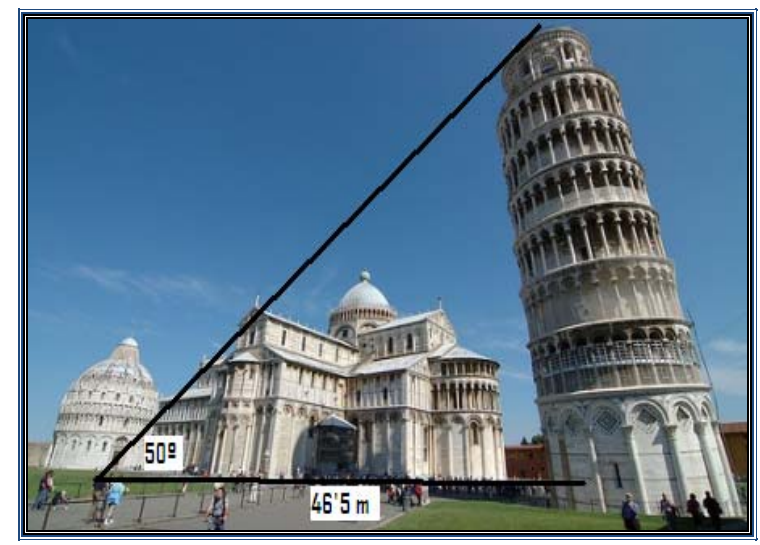

Figura 2.- Para calcular la altura de la torre.

\section{Departamentos implicados.}

Como hemos dicho, en este proyecto hay 12 departamentos implicados y entre ellos está el de matemáticas. Un ejemplo de cómo nos ayuda este proyecto en nuestra labor como profesores de matemáticas puede ser el siguiente:

\section{Matemáticas.}

Antes de la ruta.

Si nos centramos en el tema de Trigonometría para $4^{\circ}$ de ESO opción $\mathrm{B}$, una clase de matemáticas consistiría en:

- Explicación del tema

- Exposición de ejemplos

- Resolución de ejercicios y problemas
Entre los ejercicios y problemas en todos los libros de texto siempre encontramos alguno de los dos siguientes tipos:

\section{$\square$ Problema 1: Calcula la altura de la torre (figura 2).}

$\square$ Problema 2: ¿A qué distancia se encuentran los puntos A y B? (figura 3).

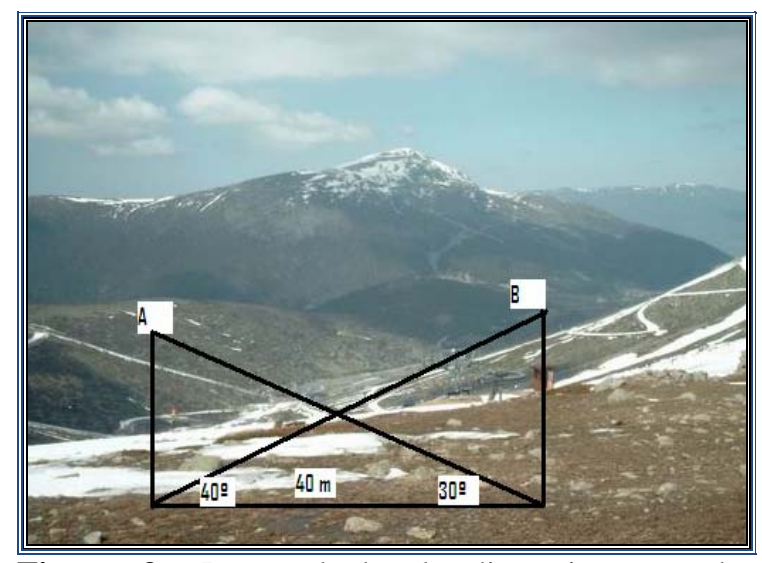

Figura 3.- Para calcular la distancia entre dos puntos.

Cuando salgamos a la ruta necesitaremos tomar medidas de diversos ángulos, para poner en práctica lo aprendido, por tanto, necesitaremos un teodolito y como en los centros educativos normalmente no los hay, entonces tendremos que fabricarlo en clase antes de la salida.

Tenemos dos posibilidades:

Forma sencilla: La longitud de la cuerda debe ser de 57 centímetros para que de esta forma la equivalencia sea $1 \mathrm{~cm}=1^{\circ}$.

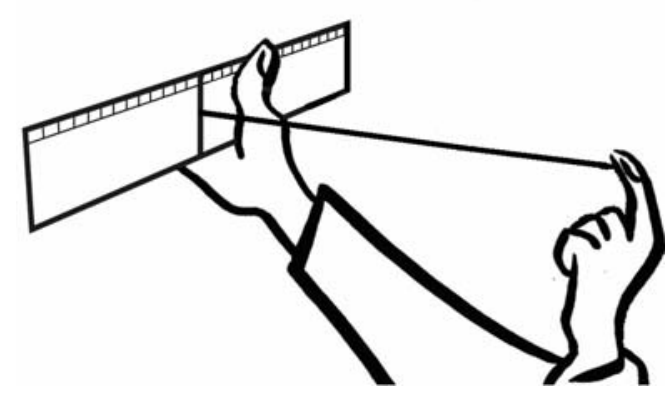

Figura 4.- Forma sencilla.

\section{Forma más complicada:}

Materiales: 


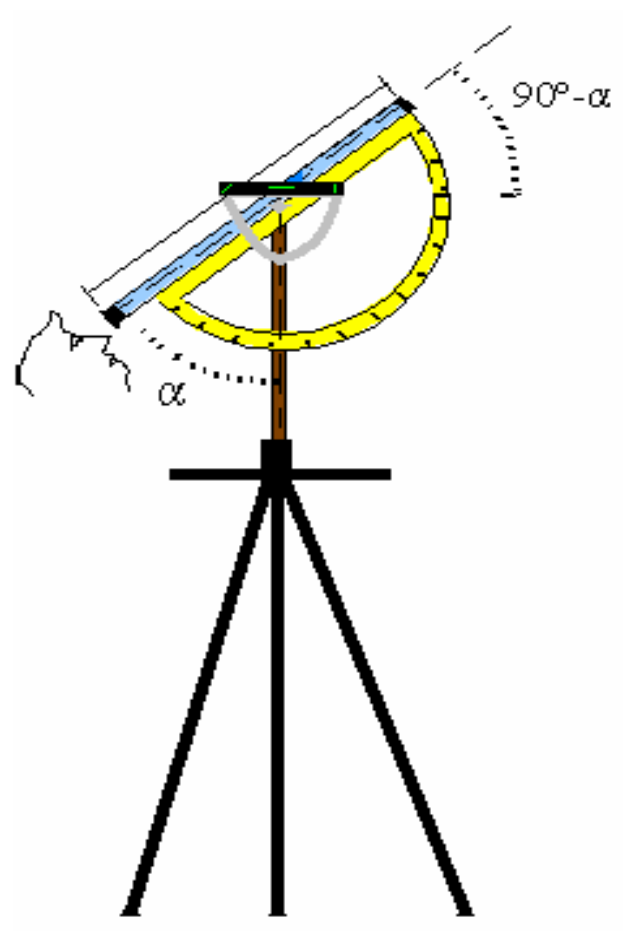

Figura 5.- Esquema del aparato para el cálculo.

- 3 palos de 1 metro de altura, pueden ser de escoba o fregona.

- Cinta adhesiva

- 3 clavos medianos

- Una tablea de marquetería

- 4 palos de unos 25 cm para soporte

- Tubo de cartón

- Hilo

- Un nivel pequeño

- Un transportador de ángulos de semicírculo y otro de circunferencia

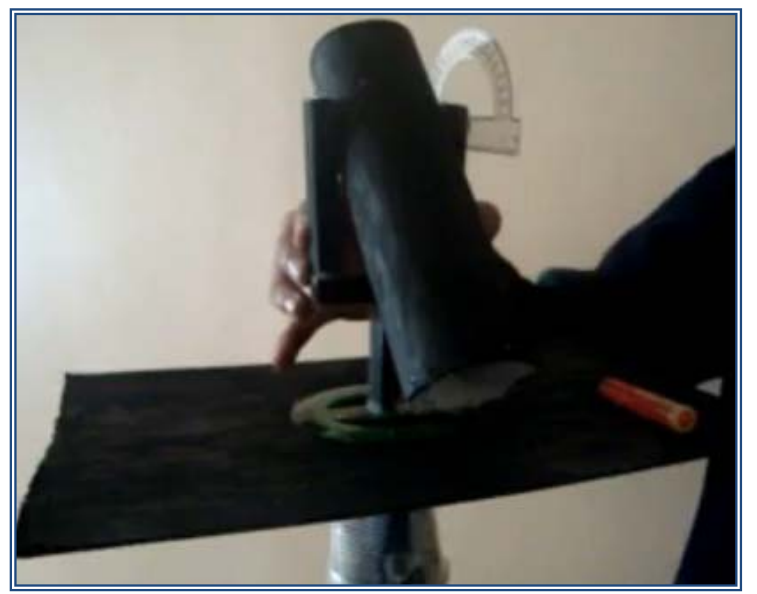

Figura 6.- Fotografía del aparato para el cálculo.
- Un plomo de pesca pequeño

Instrucciones (figura 5):

1. Con los 3 palos de 1 metro y hacemos un trípode.

2. Sobre el trípode ponemos la tabla de marquetería con la ayuda del clavo, el cual se quedará con la punta hacia arriba.

3. Colocamos, atravesado por el clavo el transportador de ángulos de circunferencia.

4. Clavamos el soporte que construimos con los palos cotos.

5. Cogemos el tubo de cartón y hacemos cuatro huecos paralelos.

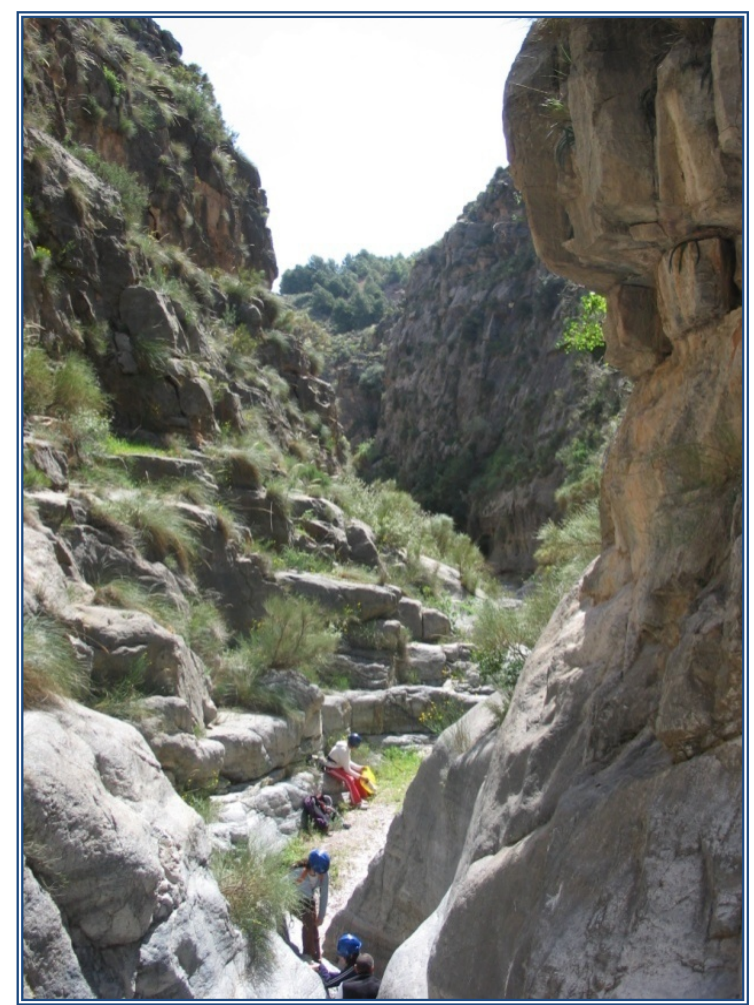

Figura 7.- Para calcular la altura del barranco.

6. Pasamos hilos, sellándolos con cinta adhesiva (mirilla).

7. Pegamos el transportador de semicírculo en el tubo con el plomo colgando de un hilo.

8. Clavamos el tubo de cartón en el soporte.

9. Por último pegamos el nivel en la marquetería. 


\section{Durante la ruta.}

En consonancia con los dos problemas expuestos a nuestro alumnado en las actividades de aula, les propondremos las siguientes actividades:

$\square$ Calcular la altura del barranco (figura 7). Necesitarán calcular ángulos, distancias, etc., necesarias para su posterior cálculo, además deberán tomar las mediciones desde tres lugares distintos.

$\square$ Calcular distancia entre pueblos (figura 8). Como desde distintos puntos de la ruta podemos ver a lo lejos diferentes poblaciones, podremos, haciendo los cálculos necesarios, conocer la distancia que hay entre ellos, por tanto tendremos que volver a medir ángulos y distancias.

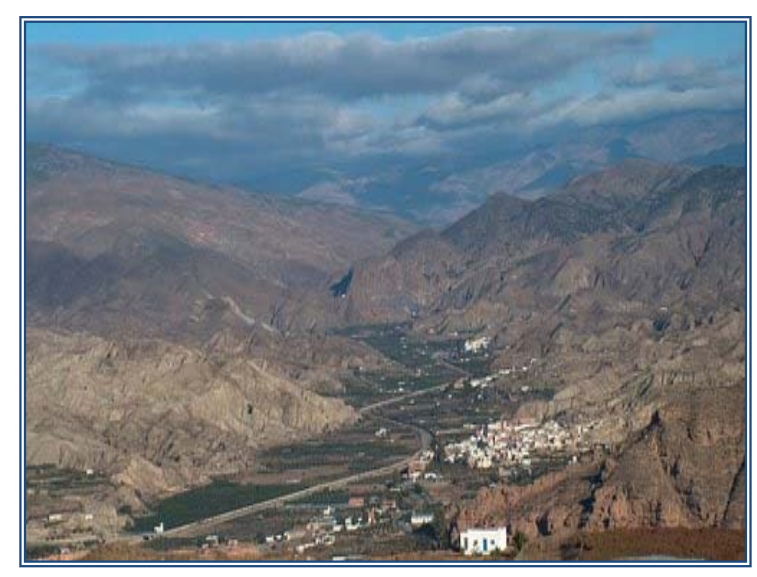

Figura 8.- Para calcular la distancia entre pueblos.

Después de la ruta.

$\mathrm{Al}$ volver tendremos material suficiente para realizar actividades en dos campos de las matemáticas, estos son:

Geometría:

- Realizamos las operaciones necesarias para resolver los problemas con las mediciones tomadas durante la ruta.

- Comparamos los aproximadamente 40 resultados obtenidos.

Estadística:

- Calculamos la media de los 40 resultados

- Calculamos la desviación típica e interpretamos el resultado
- Haciendo uso del coeficiente de variación decidimos en qué problema los resultados están más dispersos

\section{Ciencias sociales}

Ya se ha hablado del carácter multidisciplinar del Cuaderno, ya que en él se trabajan aspectos de asignaturas tan variadas como las Matemáticas, la Lengua Castellana, la Geografía, la Historia, la Biología, Geología, Física y Química, Filosofía, Música, Tecnología, e incluso Inglés y Francés y por supuesto la Educación Física. Debemos señalar en este sentido que el Cuaderno ha sido capaz de reunir al 75\% de los departamentos didácticos de los que se compone el centro.

Dentro de las Ciencias Sociales, en el campo de la Geografía, y para un nivel de $3^{\circ}$ de ESO, una actividad para realizar antes de la ruta, sería que analizasen los grandes impactos ambientales que nos podamos encontrar y que propusieran alguna medida que los pueda solucionar. Estamos comprobando cómo se pueden unir objetivos propios de la Geografía con otros propios de la Biología y/o la Ecología. La actividad sería:

$\square$ Analiza los grandes impactos ambientales que nos podamos encontrar y propón alguna medida que los pueda solucionar (tabla 1).

Tabla 1.- Los impactos ambientales.

\begin{tabular}{|l|l|}
\hline Impactos ambientales & Medidas correctoras \\
\hline & \\
\hline & \\
\hline & \\
\hline & \\
\hline & \\
\hline & \\
\hline & \\
\hline & \\
\hline
\end{tabular}

En otra de las actividades, y para realizar durante el transcurso de la ruta les pedimos que en grupos de 3 ó 4, hagan sencillas mediciones de humedad, temperatura y viento, ayudados por un higrómetro, termómetro y anemómetro, por lo que estamos trabajando a la vez, la Geografía con la Física. La actividad sería:

$\square \quad$ En grupos de 3 ó 4, haz sencillas mediciones de humedad, temperatura y viento, ayudado por un higrómetro, termómetro y anemómetro. 
LUGAR:

HORA:

DÍA:

Tabla 2.- Mediciones.

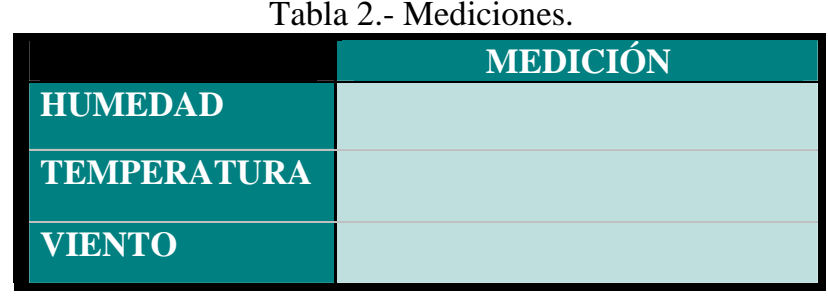

\section{Historia.}

En el caso de la Historia, y para realizar antes de la ruta, con los grupos de $4^{\circ}$ de ESO, hacemos que lean una serie de fragmentos sobre la evolución histórica de la zona antes de visitarla, por lo que estamos incentivando la lectura a través de la Historia de un medio natural que ha sido poblado por el hombre desde la Prehistoria. La actividad sería:

Lee atentamente los siguientes fragmentos sobre la evolución histórica de la zona y haz un resumen con los hitos más importantes de cada periodo:

\section{Prehistoria}

La ocupación humana de la comarca comenzó probablemente en el Paleolítico, aprovechando los inmensos bosques que cubrían entonces la Alpujarra. Durante la época neolítica estos primeros habitantes simultanearon agricultura y ganadería con la caza y recolección de frutos silvestres. En el yacimiento de los Millares se han encontrado restos de la Edad del Cobre (2500 a.C.) pertenecientes a la Cultura del mismo nombre, que representa la aparición de la metalurgia en la Península Ibérica.

\section{Colonizaciones}

La riqueza minera del Sureste peninsular atraerá el interés de los pueblos más desarrollados del Mediterráneo Oriental. Fenicios y griegos realizarán grandes aportaciones a nuestro desarrollo económico, como una agricultura más próspera, un activo comercio marítimo y una floreciente actividad pesquera con la industria de salazones. La costa se llena de factorías basadas en la comercialización del mineral extraído de la Sierra de Gádor y en la industria pesquera.

\section{Romanización}

Esta activa vida económica basada en la minería y la pesca se mantuvo durante la ocupación romana de la Bética, que era la provincia más romanizada de la Península Ibérica. Los testimonios arqueológicos son importantes. Durante los siglos III, IV y V (Bajo Imperio), Roma entra en crisis. La minería y la industria de salazones desaparecen al cerrarse el comercio mediterráneo. La costa se vuelve peligrosa ante la inseguridad y los ataques de los piratas y la población se retira a los fértiles valles del interior, más seguros y protegidos, dedicándose sobre todo a la agricultura.

\section{Musulmanes}

Esta falta de cohesión política, que continúa en los siglos siguientes, facilitó el desembarco musulmán del 711. La ocupación de la costa se reduce a una serie de torres de observación, mientras la población está dispersa en los valles del interior por una serie de alquerías o barrios autosuficientes defendidos por alcazabas y torres en las colinas. La Alpujarra se divide en tahas o divisiones administrativas creadas en época nazarí (siglo XIV), organizándose en torno a una fortaleza que servía como refugio de la población en caso de peligro y residencia del caudillo local. Nuestros vecinos de la Alpujarra se dedican a la industria textil de la seda (cultivo de morales, cría de gusanos, elaboración de seda, fabricación y comercio de tejidos...), y además se cuida el cultivo de regadío aprovechando al máximo el agua.

\section{Cristianos y moriscos}

Tras la conquista de Granada en 1492, los musulmanes quedan políticamente sometidos. Los Reyes Católicos firmarán con ellos una serie de pactos, las capitulaciones, que les permitirán quedarse, respetando su religión, leyes y costumbres. Estos pactos no se cumplen y los musulmanes se ven obligados a convertirse al cristianismo, siendo llamados moriscos. Aunque la mayor parte de la población de nuestra Alpujarra eran moriscos dedicados a una rica agricultura de regadío, el control político y económico lo posee una minoría de cristianos viejos, que viven con el temor de una rebelión de los moriscos. Prueba de este miedo y la difícil convivencia serán las torres fuertes, viviendas fortificadas dentro de las ciudades. $\mathrm{La}$ sublevación que finalmente estalla en 1568, 
provocará la expulsión de los moriscos y la posterior repoblación cristiana. Los efectos económicos serán gravísimos y la comarca entrará en una fuerte decadencia.

\section{La expansión de la uva}

El desarrollo agrícola permitió superar la profunda crisis producida por el fracaso de la minería. Por las fértiles vegas interiores se extiende el cultivo del parral y especialmente de la uva de Ohanes, cuya larga duración permite una venta en el mercado extranjero, de ahí su nombre de uva de embarque. Este monocultivo del parral atravesará crisis como la plaga de la filoxera de finales del siglo XIX o el cierre de mercados con la crisis de la Primera Guerra Mundial, pero la mantendrá como principal riqueza de la comarca hasta hace un par de décadas.

\section{La fiebre minera}

Nuestra comarca vive un temprano desarrollo económico a finales del s. XIX debido a la explotación minera. La Sierra de Gádor se agujerea de minas para extraer plomo, que era fundido para exportarlo en las numerosas y sencillas fundiciones de la propia sierra o bien en la costa, siendo la «estrella» la fundición de San Andrés de Adra, auténtica fábrica moderna donde se instalan algunas de las máquinas de vapor más antiguas de la península. Este «boom» que proporcionó trabajo a los pobres y rápidas ganancias a la burguesía, fracasó debido al pequeño tamaño de las minas, las técnicas rudimentarias, el exceso de oferta de mineral que arruinó los precios y el agotamiento de los mejores filones, dejando además la sierra desforestada, pues todos los árboles se talaron para las fundiciones.

\section{Situación actual}

Hoy en día se mantiene una fuerte contraposición entre el hábitat costero y de interior en cuanto a un desigual desarrollo económico. La ruina de las dos grandes riquezas de nuestra comarca en la época contemporánea, la minería y la uva, han dejado sin expectativas económicas los valles interiores de la comarca, mientras que la antes despoblada costa concentra la mayor parte de la población gracias a las posibilidades económicas de la agricultura bajo plástico

Son, como vemos, sólo algunos de los ejemplos que podríamos exponer. Lo que vemos aplicado en las Matemáticas o en la Geografía e
Historia es una pequeña muestra de lo que el alumnado puede realizar. Si analizásemos áreas como la Música, la Lengua, los Idiomas o cualquier otra, podríamos ver como la interdisciplinariedad está presente en cada uno de los objetivos y contenidos a través de las actividades planteadas.

\section{5.- REPERCUSIÓN SOBRE LA COMUNIDAD EDUCATIVA.}

Como conclusión podemos decir que el carácter multidisciplinar del cuaderno es incuestionable, dada la gran cantidad de departamentos didácticos implicados, pero a su vez en cada una de las actividades es evidente también su carácter interdisciplinar, tratando objetivos y métodos propios de varias materias.

De esta forma teniendo como eje vertebrador las actividades físicas en el medio natural, le damos al alumnado instrumentos y trabajamos contenidos de multitud de áreas, a través de ejercicios que se realizan antes, durante $y$ después de la ruta.

Dichos instrumentos permitirán un mayor acercamiento del alumnado a su entorno natural, así como un mejor conocimiento del mismo que le permita valorarlo en su justa medida y aprender a conservarlo y respetarlo.

Gracias a los materiales elaborados, que en breve serán publicados, se pone a disposición de los centros educativos, tanto de la zona como de otras localizaciones, una valiosa herramienta pedagógica.

\section{6.- REFERENCIAS BIBLIOGRÁFICAS.}

Aguado, A.M. (2001). Actividades Físicas en el Medio Natural en la Educación Física Escolar, colección cuadernos técnicos, $n^{\circ} 4$. Palencia: Patronato municipal de deportes.

Águila, C. (2007). Sobre el Ocio y la Posmodernidad. Un análisis sociocrítico. Sevilla: Wanceulen.

Aisenberger, B. \& Alderoqui, S. (comp). (1994). Didáctica de las Ciencias Sociales. Aportes y reflexiones. Buenos Aires: Paidós.

Altamira, R. (1985). La enseñanza de la Historia. Ed. Librería Victoriano Suárez, 2a ed.

Apostel, L. et al. (1983). Interdisciplinaridad y Ciencias Humanas. Madrid: Tecnos / UNESCO.

Baigorri, J. (1985). Elementos para una didáctica de las Ciencias Sociales. El comentario de textos históricos, la excursión geográfica y 
la utilización del vídeo. Salamanca: ICE, Grupo Cronos, Documentos Didácticos.

Beas, M. (2008). Ruta de Senderismo Nicolás Salmerón. El Eco de Alhama, 25, 106-110.

Beas, M., Martínez Moreno, A.J. \& Rodríguez Prados, F. (2009). La colaboración entre el centro educativo y otras instituciones de cara a la generación de materiales pedagógicos. "Itinerario geológico en Alhama de Almería”. II Jornadas de Prácticas de Enseñanza e Innovación Docente en el Marco del E.E.E.S. Almería: UAL.

Cuesta, R. \& Laliena, L. (coord.). (1992). ¿Cómo enseñar ciencias sociales? Aula de Innovación Educativa, núm. 8, monográfico, noviembre.
Edgardo, O. et al. (1987). El material didáctico en la enseñanza de la Historia. Ateneo. Buenos Aires.

Sánchez Bañuelos, F. (1986). Bases para una Didáctica de la Educación Física y el Deporte. Madrid: Gymnos.

Santillana Ed.. (2007). Matemáticas $4^{\circ}$ de ESO (Opción A). Proyecto La casa del saber. Madrid: Autor

Santillana Ed. (2007). Guía de recursos de Matemáticas $4^{\circ}$ de ESO (Opción A). Proyecto La casa del saber. Madrid: Autor.

Santos, M.L. (1999). Actividades físicas en el medio natural en el entorno del centro escolar de primaria. Almería: APEF, UAL.

Vicens Vives Ed. (2008). Geografía $3^{\circ}$ de ESO (Proyecto Demos). Barcelona: Autor. 